\title{
Exposición crónica a la altura. Características clínicas y diagnóstico
}

\section{Chronic exposure to altitude. Clinical characteristics and diagnosis}

\author{
Luis E. Santos-Martínez ${ }^{1,2,3^{*}}$, Ricardo A. Gómez-Tejada ${ }^{3,4}$, Carla X. Murillo-Jauregui3,5, \\ Rodrigo A. Hoyos-Paladines ${ }^{3,6}$, Carlos V. Poyares-Jardim, ${ }^{3,7}$ y Mauricio Orozco-Levi,8
}

${ }^{1}$ Departamento de Hipertensión Pulmonar y Corazón Derecho, Unidad Médica de Alta Especialidad Hospital de Cardiología, Centro Médico Nacional Siglo XXI, Instituto Mexicano del Seguro Social, Ciudad de México; ${ }^{2}$ Departamento de Cuidados Intensivos Posquirúrgicos Cardiovasculares, Secretaría de Salubridad y Asistencia, Instituto Nacional de Cardiología Ignacio Chávez, Ciudad de México, México; ${ }^{3}$ Departamento de Circulación Pulmonar, Asociación Latinoamericana del Tórax; ${ }^{4}$ División de Neumología, Hospital de Clínicas José de San Martín, Facultad de Medicina, Universidad de Buenos Aires, Buenos Aires, Argentina; ${ }^{5}$ Unidad de Fisiología y Fisiopatología Respiratoria, Instituto Boliviano de Biología de Altura, La Paz, Bolivia; ${ }^{6}$ Clínica de Hipertensión Pulmonar, Hospital Carlos Andrade Marín, Instituto Ecuatoriano de Seguridad Social, Quito, Ecuador; ${ }^{7}$ Divisao de Pneumologia, Instituto do Coracao, InCor/HCFMUSP (Hospital das Clinicas da Universidade de Sao Paulo), Sao Paulo, Brasil; ${ }^{8}$ Servicio de Neumología, Hospital Internacional de Colombia, Fundación Cardiovascular de Colombia, Floridablanca, Colombia

\section{Resumen}

La exposición crónica a la altitud se ha asociado a hipoxia hipobárica en quienes la experimentan. Dos entidades se han asociado a la hipoxia hipobárica: la hipertensión pulmonar de la alta altitud y el mal de montaña crónico. Se describen sus características fisiológicas y de la circulación pulmonar, así como su perfil clínico y el diagnóstico.

Palabras clave: Circulación pulmonar. Hipertensión pulmonar. Altitud. Mal de montaña crónica. Hipoxia crónica.

\begin{abstract}
Chronic exposure to altitude has been associated with hypobaric hypoxia in its inhabitants. Two entities have been associated with it, high altitude pulmonary hypertension and chronic mountain sickness. Its physiological and pulmonary circulation characteristics are described, as well as its clinical profile and diagnosis.
\end{abstract}

Key words: Pulmonary circulation. Pulmonary hypertension. Altitude. Chronic mountain sickness. Chronic hypoxia.

\section{Introducción}

Alrededor de 140 millones de personas viven en altitudes > 2,500 metros sobre el nivel del mar (msnm). Altas densidades poblacionales se encuentran establecidas a más de 3,500 msnm. Para vivir a estas alturas se han requerido largos periodos de tiempo para adaptarse por medio de cambios anatómicos y fisiológicos del corazón y de la circulación pulmonar, entre otros ${ }^{1}$. Por lo que en razón de estos cambios se ha categorizado la altitud de acuerdo con los msnm en: 1) altitud moderada, 1,500-2,500 msnm (no se sienten efectos en ejercicio); 2) altitud alta, 2,500-3,500 msnm

\section{Correspondencia:}

*Luis E. Santos-Martínez

E-mail: luis.santos@cardiologia.org.mx
Fecha de recepción: 02-10-2020

Fecha de aceptación: 18-01-2021

DOI: 10.24875/ACM.20000447
Disponible en internet: 25-03-2021

Arch Cardiol Mex. (ahead of print) www.archivoscardiologia.com

1405-9940 / @ 2021 Instituto Nacional de Cardiología Ignacio Chávez. Publicado por Permanyer. Este es un artículo open access bajo la licencia CC BY-NC-ND (http://creativecommons.org/licenses/by-nc-nd/4.0/). 
(se sienten efectos en ejercicio); 3) altitud muy alta, 3,500-5,800 msnm (se sienten efectos hasta en reposo), y 4) altitud extremadamente alta, $>5,800 \mathrm{msnm}$ (la vida permanente parecería imposible) $)^{2,3}$.

Las poblaciones de la alta altitud (HA, por sus siglas en inglés, high altitude) realizan sus actividades cotidianas sin evidencia de afección alguna; se encuentran diseminadas en cuatro grandes áreas: los Andes en América del Sur, las Montañas Rocosas en EE.UU., Etiopía en África y la región de los Himalaya en $\mathrm{Asia}^{2,3}$. De estas áreas, las poblaciones más antiguas de habitantes de altitudes $>2,500 \mathrm{msnm}$ son las del Tíbet y los sherpas en Asia, y los etíopes en África. El perfil clínico de los tibetanos exhibe mayor saturación arterial de oxígeno $\left(\mathrm{SaO}_{2}\right)$, menor hemoglobina $(\mathrm{Hb})$ y mayor capacidad ventilatoria que los habitantes de Han (China) y los nativos andinos. Esto sugiere que lograron una adaptación propia a la altura por selección natural a lo largo de miles de años y numerosas generaciones ${ }^{1,4}$.

En América, los aymaras y los quechuas (Andes) son poblaciones longevas con más de 11,000 años de existencia, que aún están en el lento proceso de adaptación. Su perfil clínico es de niveles elevados de $\mathrm{Hb}$, menor $\mathrm{SaO}_{2}$, aumento de la presión arterial pulmonar (PAP) y resistencia vascular pulmonar (RVP), con hipertrofia del ventrículo derecho (VD) y aumento de volúmenes pulmonares. Desde esta perspectiva, los tibetanos se han adaptado genotípicamente, en tanto que los nativos andinos se ubican en el proceso de adaptación fenotípica dado su menor tiempo de residencia en la altitud ${ }^{5,6}$.

Además de las personas que viven en estas alturas, se ha estimado que alrededor de 40 millones que habitan a menor altitud viajan cada año a regiones de HA y mayores con fines de turismo, trabajo, militares y de rescate $^{2,3}$. Con frecuencia estas personas no tienen tiempo para aclimatarse y en otras ocasiones hay pérdida de la adaptación en quienes son nativos de la altitud ${ }^{4,6}$.

Qué tan bien esta población puede adaptarse a la HA depende de la frecuencia de ascenso a la altura, la velocidad de ascenso, la severidad y la duración de su exposición y su genética ${ }^{2,7,8}$.

La mala adaptación puede ocurrir en condición aguda o crónica. Existen variaciones significativas de la respuesta de las personas a la vasoconstricción pulmonar hipóxica (VPH). Los llamados respondedores activos (0 hiperreactores) pueden presentar mala adaptación aguda al aumento de la PAP y desarrollar edema pulmonar, mal de montaña agudo y edema cerebral ${ }^{8,9}$.
En otro escenario, luego de periodos largos en la altura, otras personas, muestran inadecuada adaptación que se manifiesta por insuficiencia cardiaca secundaria a hipertensión pulmonar (HP) severa. Al ser una respuesta a la VPH, este escenario de la HP puede ocurrir en condición aguda o asociada a exposición crónica a la altitud ${ }^{2}$. También pueden ocurrir síndromes de eritrocitosis excesiva o mal de montaña crónico $(\mathrm{MMC})^{2,10-14}$. Son enfermedades de la circulación pulmonar de curso potencialmente fatal que expresan una inadecuada respuesta adaptativa a la hipoxia crónica en la altitud.

En este trabajo describiremos los rasgos fisiológicos y de la circulación pulmonar en la altitud, así como la clínica y el diagnóstico de las dos entidades principales crónicas de la circulación pulmonar en la altitud: la HP asociada a la alta altitud (HPHA) y el MMC o enfermedad de Monge. Cabe acotar que el Profesor Carlos Monge (Perú) fue el primero en realizar su descripción clínica.

\section{Consideraciones fisiológicas de la altitud}

Las personas que nacen (nativos) y que continúan viviendo en la altitud son sanos, aunque durante toda su vida están expuestos a la hipoxia hipobárica que conduce a hipoxia crónica. Las personas expuestas a la $H A$ requieren adaptarse a este ambiente hipóxico hipobárico de la altura. Por arriba de los 2,500 msnm, la presión barométrica y la presión inspirada de oxígeno disminuyen y el resultado es hipoxia alveolar e hipoxemia, lo que deriva en VPH, esto es, se eleva en grado moderado la PAP y la del VD, pero es compatible con una vida normal en $\mathrm{HA}^{5,8,9}$. La VPH permite un mejor equilibrio entre la relación ventilación/perfusión $(\mathrm{V} / \mathrm{Q})$ pulmonar y aumenta la capacidad de difusión pulmonar; en comparación con el nativo a nivel del mar, se traduce en un menor gradiente alveoloarterial de oxígeno $(\mathrm{A}-\mathrm{a}=3 \text { a } 5 \mathrm{mmHg})^{1,5,15}$.

Al estimular la eritropoyesis la hipoxemia promueve que se incrementen los niveles de eritrocitos y de $\mathrm{Hb}$, lo que permite que se adquiera mayor capacidad para transportar oxígeno $0^{1,5,12-15}$. La finalidad de estos ajustes funcionales es distribuir la sangre a todos los segmentos pulmonares y hacer más eficiente el transporte de oxígeno.

Otras diferencias que se han observado en el nativo de la altitud es que tiene menor ventilación/minuto y menor respuesta de los quimiorreceptores periféricos (carotídeo y aórtico) a la presión arterial de oxígeno $\left(\mathrm{PaO}_{2}\right)$ y al bióxido de carbono $\left(\mathrm{PaCO}_{2}\right)$ que el 
observado en sujetos nativos del nivel del mar ${ }^{15}$, lo que está relacionado con la génesis de la hipercapnia y en forma secundaria a la hipoxemia. La variación de la ventilación pulmonar es diferente en respuesta al reto con oxígeno: a nivel del mar es del $10 \%$ vs. el $30 \%$ en personas del nivel del mar aclimatadas a la altura vs. el $15 \%$ en residentes nativos de la altitud. Esto explica por qué las personas nativas del nivel del mar que ascienden a la HA tienen hiperventilación como respuesta compensatoria a la hipoxia ${ }^{15}$, y no es causada por alteraciones en la distensibilidad pulmonar, ya que esta es similar en nativos del nivel del mar y de la altitud (160 a $220 \mathrm{ml} / \mathrm{cmH}_{2} \mathrm{O}$ para la ciudad de la $\mathrm{Paz}$, Bolivia, 3,630 msnm). Las adaptaciones de la caja torácica en la altitud han permitido un incremento de 1.8 $\mathrm{cm}$ en el diámetro anteroposterior del tórax y en los volúmenes pulmonares: capacidad vital forzada $384 \mathrm{ml}$ y en el volumen residual del $13 \%$, con disminución de la capacidad residual funcional del $11 \%{ }^{15}$.

Otra consideración en la HA es que sus habitantes viven en un medio ambiente diferente al del nivel del mar: la temperatura y la humedad disminuyen, y el viento y la radiación solar son mayores (un medio ambiente con tendencia a la sequedad) $)^{1,15}$.

\section{Características de la circulación pulmonar y del corazón derecho en la altitud}

La característica clínica de la circulación pulmonar y corazón derecho del nativo normal de la HA es la HP con hipertrofia del VD, e incremento del grosor de la capa de células musculares lisas (CML) de la arteria pulmonar. Estas hallazgos se vuelven exagerados cuando este nativo sano pierde su capacidad de adaptación a su altitud y desarrolla eritrocitosis excesiva y MMC1.

En nativos de la HA y a nivel del mar, en la vida intrauterina y hasta el momento del parto, se describe por igual un patrón fetal de la circulación pulmonar que se caracteriza por: HP y aumento del grosor de la capa de CML con disminución de la luz vascular, además de hipertrofia del VD. A diferencia de los parámetros observados a nivel del mar, donde ocurre normalización de estos en los primeros 4 a 6 meses de vida, en los nacidos en la HA los cambios posnatales persisten en la PAP, en la estructura de las pequeñas arterias pulmonares y en el VD. En estas poblaciones la PAP media es de alrededor de $60 \mathrm{mmHg}$ y es similar a nivel del mar y en la HA. Hacia las 72 horas de vida la PAP media disminuye a 55 y $45 \mathrm{mmHg}$ en los primeros 1 a 5 años de vida. En adolescentes y adultos se mantiene en $28 \pm 10.5 \mathrm{mmHg}$. La RVP en HA se ha obtenido en $332 \pm 212.6 \mathrm{HA}$ vs. $69 \pm 25.3$ dyn.s. $\mathrm{cm}^{-5}$ a nivel del mar. En adultos a nivel del mar, una vez la PAP media desciende a las 72 horas, esta se mantiene alrededor de $12 \pm 2.2 \mathrm{mmHg}$ (estimada en adultos de 17 a 23 años). En ambas poblaciones estudiadas, HA y a nivel del mar, los valores hemodinámicos como la presión de aurícula derecha (PAD), gasto cardiaco, índice cardiaco y la presión capilar pulmonar se observaron normales $^{1,5-7}$. La elevada PAP media en la HA al nacimiento provoca el retardo del cierre del conducto arterioso.

\section{Correlación de la altitud con la presión arterial pulmonar, saturación arterial de oxígeno y hemoglobina}

El nivel de altitud tiene una correlación inversa con la $\mathrm{SaO}_{2}$ y directa con la PAP. El impacto de la altitud en estos parámetros es evidente a partir de los $3,000 \mathrm{msnm}$. A $4,540 \mathrm{msnm}$ la $\mathrm{SaO}_{2}$ es $78.4 \pm 4.93 \%$ vs. $94.91 \pm 2.12$ a nivel del $\operatorname{mar}(p<0.001)$. La Hb tiende a incrementar a $19.5 \pm 1.97$ vs. $14.7 \pm 0.88 \mathrm{~g} / \mathrm{dl}$ a nivel del mar $(p<0.001)^{1,5,12,13}$.

Es de considerar que la PAP exhibe variabilidad importante, intersujetos e interespecies. En la HA la PAP media fue mayor a $25 \mathrm{mmHg}$, y un $10 \%$ fue $>40 \mathrm{mmHg}$, sin embargo, el $10 \%$ tuvieron PAP media normal. De acuerdo con el estímulo hipóxico, hay sujetos hiporreactivos e hiperreactivos, que parecen estar condicionados por la susceptibilidad genética ${ }^{8,16}$, por medio de mediadores de vías de señalización intracelular a la hipoxia crónica como el factor 1 inducido por hipoxia ${ }^{17}$. Esto explicaría por qué algunos recién nacidos y lactantes son hiperreactivos a la hipoxia de la HA y llegan a desarrollar HP severa e insuficiencia cardiaca por el aumento de grosor de la capa de CML en las pequeñas arterias pulmonares.

De acuerdo con estas características, la HP y el MMC son entidades similares que comparten la exposición crónica a la altitud ${ }^{1,5,6}$ y sus perfiles clínicos para el diagnóstico.

\section{Hipertensión pulmonar asociada a la alta altitud}

Es un síndrome que ocurre en niños y adultos que residen por arriba de 2,500 msnm. De acuerdo con el Consenso de Enfermedades Subagudas y Crónicas de la Alta Altitud publicado en el año $2005^{18}$, este síndrome se caracteriza por tener PAP media $>30 \mathrm{mmHg} 0$ 
PAP sistólica $>50 \mathrm{mmHg}$ obtenida en su lugar de residencia en la altitud; además de hipertrofia del VD, insuficiencia cardiaca, hipoxemia moderada y ausencia de eritrocitosis excesiva, definida como un valor de $\mathrm{Hb}$ $<19 \mathrm{~g} / \mathrm{dl}$ en mujeres $\mathrm{y}<21 \mathrm{~g} / \mathrm{dl}$ en hombres.

\section{Síntomas}

La presentación clínica en etapas tempranas suele ser asintomática, posteriormente se hace evidente en ejercicio y al final en reposo. El síntoma más frecuente es la disnea. En la evolución pueden sumarse fatiga, debilidad, mareos y síncope, dolor de tórax tipo anginoso e intolerancia al ejercicio. Se pueden referir, además: cefalea, mareos, insomnio, somnolencia, alteraciones cognitivas, confusión y deterioro de la memoria ${ }^{18,19}$.

\section{Examen físico}

Hallazgos relacionados al compromiso vascular pulmonar: a) segundo ruido cardiaco intenso a la auscultación (cierre brusco de la válvula pulmonar, 2P); b) expansión torácica palpable sobre el margen inferior izquierdo del esternón (VD dilatado); c) auscultación de soplo holosistólico de regurgitación (incompetencia de la válvula tricúspide), y d) puede haber episodios de insuficiencia cardiaca derecha y signos de congestión venosa sistémica (ingurgitación yugular, reflujo hepatoyugular, hepatomegalia, ascitis, edema de miembros inferiores), que podrían superponerse con signos de insuficiencia ventricular izquierda ${ }^{14,17}$. Estos signos pueden ser más evidentes en chinos de la etnia Han que incluso en residentes de la HA de los Andes ${ }^{1,17}$.

\section{Estudios complementarios ${ }^{20-22}$}

\section{ELECTROCARDIOGRAMA}

El electrocardiograma (ECG) está ampliamente disponible, pero posee baja sensibilidad diagnóstica. Son significativos: a) onda $\mathrm{P}$ pulmonale ( $>3 \mathrm{~mm}$ en $\mathrm{D}_{2}, \mathrm{D}_{3}$, aVF); b) rotación del eje QRS a la derecha; c) bloqueo de rama derecha; $d$ ) $R / S>1$ en $V_{1}$ (probable hipertrofia del VD), y e) descenso segmento ST desde $V_{1}$ a $V_{3}$.

\section{RADIOGRAFÍA DE TÓRAX}

Es ineludible ante pacientes con disnea, dolor torácico o síntomas respiratorios. Tiene limitada sensibilidad diagnóstica para HP. En HP avanzada puede observarse: a) agrandamiento ventricular derecho; b) arco medio cardiaco prominente, y c) distribución atípica de la circulación pulmonar.

\section{Pruebas de función Respiratoria}

Las pruebas de función respiratoria (PFR) permiten excluir afecciones respiratorias que en distinto grado se superponen con HPHA. La enfermedad pulmonar obstructiva crónica (EPOC) y la enfermedad pulmonar intersticial y fibrosis (EPI) se asocian con HP de grupo 3. Las PFR cuantifican el compromiso de la función pulmonar y ofrecen criterios de evolución. En la HPHA las PFR suelen ser normales ${ }^{22,23}$.

\section{ECOCARDIOGRAFÍA}

Es el estudio característico de tamizaje en HP y puede indicar: a) incremento de la PAP; b) aumento del tamaño del ventrículo y aurícula derechos, y c) alteración de la función del ventrículo izquierdo (VI). Tiene mejor sensibilidad y especificidad que el ECG, pero puede subestimar o sobreestimar la medición de la PAP. Ambos estudios no invasivos (ECG y ecocardiografía) se han combinado entre sí para la detección de $\mathrm{HPHA}^{21,22}$. La PAP sistólica se calcula agregando la presión de la aurícula derecha (AD) al gradiente de presión entre el VD y la $A D$, con la fórmula: $\nabla P-V D / A D$, utilizando la ecuación modificada de Bernouilli: $\nabla P-V D /$ $A D=4 V^{2}$, donde $V$ es la velocidad pico del jet regurgitante a través de la válvula tricuspídea y $\nabla P$ es el gradiente de presión. Diversos estudios en altitud se han realizado con ecocardiografía dada la mayor factibilidad para disponer de equipos en esas altitudes, lo que explica el punto de corte de la PAP sistólica.

\section{Gammagrafía dE VENTILACIÓN/PERFUSIÓN}

La HP asociada a tromboembolia pulmonar crónica (HPTEC) o HP de grupo 4 debe ser descartada como norma en el diagnóstico de enfermedades vasculares pulmonares. El gammagrama $\mathrm{V} / \mathrm{Q}$ puede demostrar amplios defectos de perfusión respecto de la ventilación llamados desigualdad $\mathrm{V} / \mathrm{Q}^{24}$. Estos defectos indican la presencia de trombos (coágulos) en la circulación pulmonar, pero su sensibilidad y especificidad diagnóstica disminuyen en presencia de enfermedad pulmonar previa. También pueden observarse defectos de perfusión en otras patologías que condicionan desigualdad V/Q: sarcoidosis, sarcoma, vasculitis y émbolos diferentes a coágulos como en la esquistosomiasis ${ }^{21,25}$. 
Tabla 1. Clasificación de la hipertensión pulmonar de la Organización Mundial de la Salud 2018, Niza, Francia

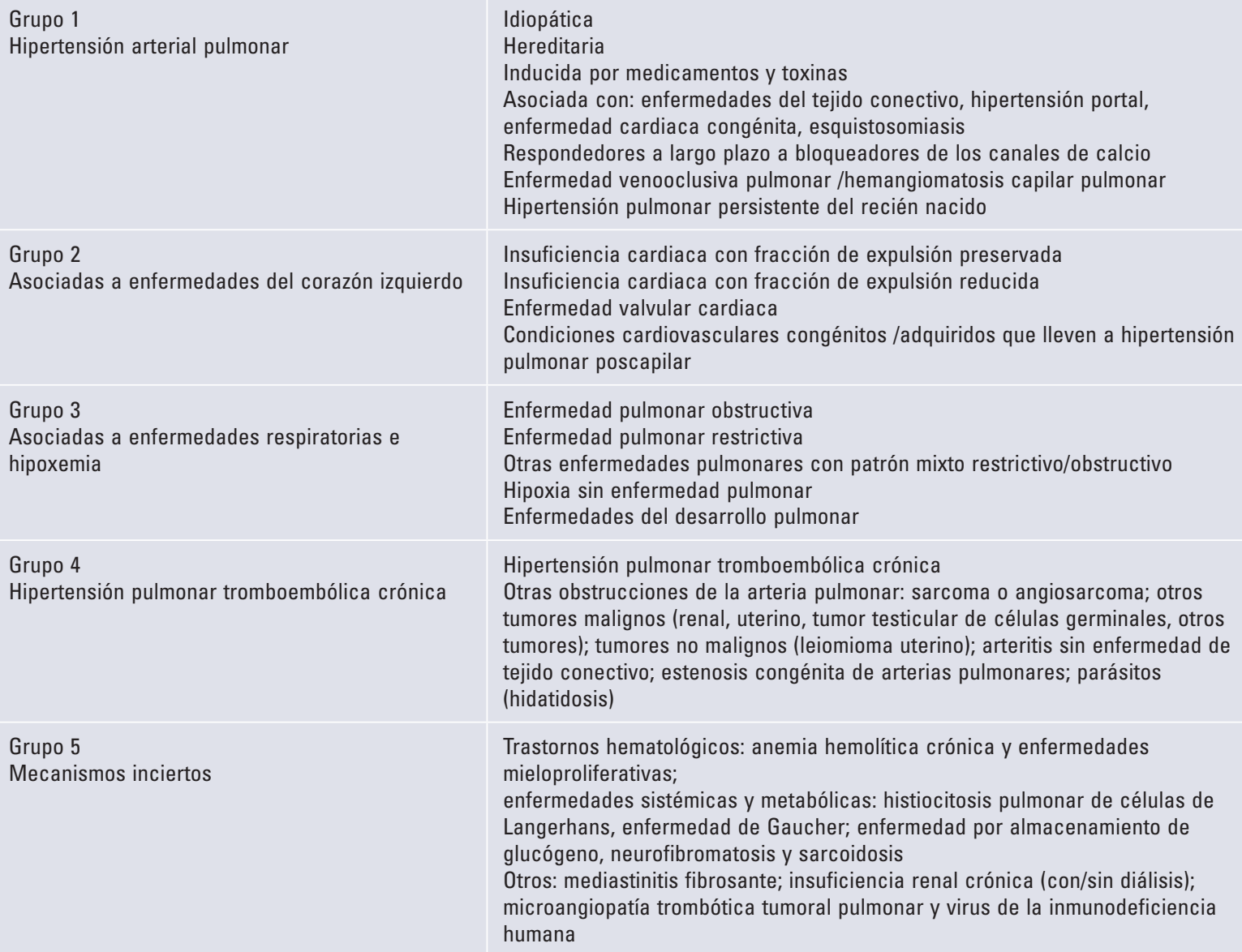

Enfermedad pulmonar obstructiva

Enfermedad pulmonar restrictiva

Otras enfermedades pulmonares con patrón mixto restrictivo/obstructivo

Hipoxia sin enfermedad pulmonar

Enfermedades del desarrollo pulmonar

Hipertensión pulmonar tromboembólica crónica

Otras obstrucciones de la arteria pulmonar: sarcoma o angiosarcoma; otros tumores malignos (renal, uterino, tumor testicular de células germinales, otros tumores); tumores no malignos (leiomioma uterino); arteritis sin enfermedad de tejido conectivo; estenosis congénita de arterias pulmonares; parásitos (hidatidosis)

Trastornos hematológicos: anemia hemolítica crónica y enfermedades mieloproliferativas;

enfermedades sistémicas y metabólicas: histiocitosis pulmonar de células de Langerhans, enfermedad de Gaucher; enfermedad por almacenamiento de glucógeno, neurofibromatosis y sarcoidosis

Otros: mediastinitis fibrosante; insuficiencia renal crónica (con/sin diálisis); microangiopatía trombótica tumoral pulmonar y virus de la inmunodeficiencia humana

\section{Angiotomografía pulmonar}

Es necesaria para excluir los diagnósticos diferenciales de la HP de grupo 4, así como para cuantificar la severidad y extensión del proceso tromboembólico crónico. En pacientes con gammagrama $\mathrm{V} / \mathrm{Q}$ de alta probabilidad para HPTEC deberá preferirse a la angiografía pulmonar tradicional ${ }^{21,22,25}$.

\section{Cateterismo cardiaco derecho}

El cateterismo cardiaco derecho (CCD) es el diagnóstico de certeza en HPHA, como de otras formas de HP que comparten la característica de tener componente precapilar ${ }^{26}$. En la HA, la HP se define mediante el registro de la PAP media $>30 \mathrm{mmHg}$ o PAP sistólica $>50 \mathrm{mmHg}$, que deberán considerarse como criterios específicos para esta forma de HP asociada a la altitud 5 ,18.

De forma más general la definición hemodinámica de la HP precapilar del Sexto Simposio Mundial de la
HP de la Organización Mundial de la Salud (OMS) del año 2018 realizado en Niza, Francia ${ }^{26}$, consigna para el diagnóstico de HP que se requiere tener una PAP media $>20 \mathrm{mmHg}$, presión de oclusión pulmonar $\leq 15 \mathrm{mmHg}$ y RVP $\geq 3$ unidades Wood. La presión de oclusión pulmonar ha de medirse con extremo cuidado, dado que los valores $\geq 15 \mathrm{mmHg}$ orientan al diagnóstico de HP grupo 2 (enfermedades asociadas al VI). La evaluación hemodinámica deberá complementarse con medición de la presión de fin de diástole del VI, si se sospecha HP asociada a cardiopatía del $\mathrm{V}^{26}$. En la clasificación de la HP de la OMS, la HPHA se encuentra categorizada en el grupo 3: HP debida a enfermedades pulmonares y/o hipoxia (Tabla 1) ${ }^{26,27}$.

Estos dos niveles de corte para la PAP complican actualmente el diagnóstico de HPAH y el conocer una prevalencia verdadera, sin embargo, los dos señalan la existencia de una forma diferente de elevación de la PAP y es debida a la hipoxia en condiciones de baja presión atmosférica. Se requiere la unificación de 
criterios. La prevalencia en realidad se desconoce debido a que la PAP no es sistemáticamente examinada. Se han utilizado signos subrogados (proxy) para estimarla. Aldashev, et al. ${ }^{20,21}$ la estimó de 741 personas habitantes permanentes de la altitud mediante la obtención de signos del ECG de cor pulmonale, 23\% hombres y $6 \%$ mujeres. La PAP media obtenida por CCD fue de $31.6 \pm 3.9$ (rango: 20 a $64 \mathrm{mmHg}$ ).

Para aumentar estas áreas de incertidumbre en un metaanálisis ecocardiográfico reciente de Soria, et al. ${ }^{28}$, donde se estudiaron 287 sujetos ( 9 estudios) con MMC en reposo y 142 (5 estudios) en ejercicio, se señaló que la PAP media fue en promedio $18 \mathrm{mmHg}$ en reposo y $31 \mathrm{mmHg}$ en ejercicio, lo que sugirió la no existencia de HP en reposo y ser ligera en ejercicio. Esta información hace que la elevación de la PAP en altitud en reposo parezca endeble ${ }^{29}$, sin embargo, estudios futuros con CCD darán más información al respecto.

\section{FACTORES DE RIESGO}

Historia de HPAH, historia de vasoconstricción pulmonar excesiva persistente en respuesta a la hipoxia, hipoxemia durante el sueño ${ }^{18}$.

Deben buscarse intencionadamente comorbilidades que pueden agravar la condición clínica u otras entidades del mismo grupo $3^{23,26}$ asociadas a la HP, como EPOC, EPI, síndromes de apnea obstructiva del sueño (SAOS) y escleroderma (Tabla 1), mismas que al estar presentes descartarían el diagnóstico de HPHA ${ }^{18,23}$.

\section{Resumen}

El diagnóstico de HPHA depende de datos epidemiológicos relacionados a la exposición crónica a la altura junto a datos clínicos compatibles. Se sumarán estudios auxiliares que permitan descartar la pertenencia a los diferentes grupos de HP señalados en la tabla 1. El CCD es la herramienta principal para establecer el diagnóstico hemodinámico, y hasta unificarse los criterios se deberán utilizar los del Consenso de la Altitud ${ }^{18}$.

\section{Mal de montaña crónico. Enfermedad de Monge}

EI MMC posee diferentes expresiones clínicas. En inmigrantes y nativos del Himalaya, expuestos en forma prolongada a HA se suele presentar insuficiencia cardiaca congestiva con escasa o moderada eritrocitosis. Por su parte, en zonas andinas de Perú y Bolivia los pacientes reproducen un síndrome respiratorio de hipoventilación alveolar ${ }^{1,5,15}$ caracterizado por severa hipoxemia e hipoventilación con hipercapnia. Se acompaña de eritrocitosis excesiva $(\mathrm{Hb}>19 \mathrm{~g} / \mathrm{dl}$ en mujeres y $\mathrm{Hb}>21 \mathrm{~g} / \mathrm{dl}$ en varones), HP moderada a severa $\mathrm{y}$ evolución potencial a cor pulmonale con insuficiencia cardiaca congestiva. La diferencia del MMC con respecto a la HPHA la constituye la eritrocitosis excesiva ${ }^{18}$.

\section{Síntomas}

Cefalea, mareos, tinnitus, disnea, palpitaciones, pérdida del apetito, tolerancia al ejercicio disminuida, parestesias y enrojecimiento de palmas y plantas de los pies, fatiga física y mental, concentración mental deteriorada, alteraciones del sueño, dolor muscular y de articulaciones. Suelen revertirse por traslado a nivel del mar y la sintomatología reinicia al regresar la persona a la $\mathrm{HA}^{10,30}$.

\section{Signos}

Eritrocitosis excesiva, hipoxemia severa, cianosis localizada, dilataciones venosas, acropaquia (dedos en palillo de tambor) en manos y pies, incremento de la tensión arterial diastólica (asociado a la eritrocitosis). Auscultación del segundo ruido pulmonar aumentado (2P) y soplo mesosistólico eyectivo. Pueden presentarse signos moderados de insuficiencia cardiaca y coexistir con $\mathrm{HP}^{10,30}$.

\section{Estudios complementarios ${ }^{10,15,18,30}$}

\section{RADIOGRAFÍA DE TÓRAX}

Puede observarse: a) cardiomegalia; b) prominencia de la arteria pulmonar en el borde cardiaco izquierdo, y c) acentuación de las marcas vasculares en el centro y periferia de los campos pulmonares.

\section{ELECTROCARDIOGRAMA}

Puede observarse: a) ondas P en pico en DII, DIII, aVF; b) rotación del eje de QRS hacia la derecha; c) patrón $r S$ en precordiales derechas, y d) ondas $T$ negativas en precordiales derechas, debida a sobrecarga del VD.

\section{Pruebas de función RESPIRATORIA}

La forma clásica o primaria del MMC debe contar con PFR normales y se deben excluir otras causas secundarias de hipoxemia como determinantes de 
Tabla 2. Escala de Qinghai para mal de montaña crónico

\begin{tabular}{|c|c|}
\hline $\begin{array}{l}\text { Falta de aliento y/o } \\
\text { palpitaciones }\end{array}$ & $\begin{array}{l}0 \text { No } \\
1 \text { Leve } \\
2 \text { Moderada } \\
3 \text { Severa }\end{array}$ \\
\hline Trastornos del sueño & $\begin{array}{l}0 \text { Dormir usual } \\
1 \text { No duerme como lo usual } \\
2 \text { Despierta muchas veces/sueño } \\
\text { nocturno pobre } \\
3 \text { No puede dormir nada }\end{array}$ \\
\hline Cianosis & $\begin{array}{l}0 \text { No } \\
1 \text { Leve } \\
2 \text { Moderada } \\
3 \text { Severa }\end{array}$ \\
\hline Dilatación de las venas & $\begin{array}{l}0 \text { No } \\
1 \text { Leve } \\
2 \text { Moderada } \\
3 \text { Severa }\end{array}$ \\
\hline Parestesias & $\begin{array}{l}0 \text { No } \\
1 \text { Leve } \\
2 \text { Moderada } \\
3 \text { Severa }\end{array}$ \\
\hline Cefalea & $\begin{array}{l}0 \text { No } \\
1 \text { Leve } \\
2 \text { Moderada } \\
3 \text { Severa }\end{array}$ \\
\hline Tinnitus & $\begin{array}{l}0 \text { No } \\
1 \text { Leve } \\
2 \text { Moderada } \\
3 \text { Severa }\end{array}$ \\
\hline Hemoglobina & $\begin{array}{l}\text { Masculino: } 0>18 \mathrm{~g}<21 \mathrm{~g} / \mathrm{dl} \\
3 \geq 21 \mathrm{~g} / \mathrm{dl} \\
\text { Femenino: } 0>16 \mathrm{~g}<19 \mathrm{~g} / \mathrm{dl} \\
3 \geq 19 \mathrm{~g} / \mathrm{dl}\end{array}$ \\
\hline
\end{tabular}

eritrocitosis: EPOC, fibrosis quística, EPI, bronquiectasias, trastornos del dormir, etc.

\section{Datos significativos del CATETERISMo CARDIACO DERECHO}

Peñaloza y Arias Stella ${ }^{1}$ han relacionado los hallazgos de la hemodinámica entre pacientes con MMC y residentes de la HA sanos. Encontraron diferencias significativas para PAP media y RVP, así como de los valores del hematocrito, $\mathrm{Hb}$ y $\mathrm{SaO}_{2}$. No hallaron diferencias en la PAD, presión capilar pulmonar o en el índice cardiaco. Datos similares han sido obtenidos por otros ${ }^{15}$.

\section{Escala de gravedad Qinghal del mal de MONTAÑA CRÓNICO ${ }^{18}$}

Evalúa la severidad del MMC. Se utiliza también para fines de comparación entre casos individuales de MMC y los que ocurren entre países.
La severidad del MMC puede ser definido de acuerdo con la suma de puntos dados a cada síntoma y a la $\mathrm{Hb}$ : ausente $0-5$ puntos, leve 6-10 puntos, moderado 11-14 puntos y severo $>15$ puntos (Tabla 2).

\section{FACTORES dE RIESGO PARA MAL DE MONTAÑA CRÓNICO}

Los siguientes son factores de riesgo para el MMC: a) historia previa de la enfermedad; b) SAOS y otras hipopneas; c) historia de alteraciones de la sensibilidad a la hipoxia e hipoventilación; d) sobrepeso/obesidad, y e) estados posmenopáusicos.

\section{Resumen}

El diagnóstico de MMC dependerá de datos clínicos sugestivos, epidemiología de la enfermedad, así como de los resultantes de estudios complementarios que permitan descartar o confirmar otras entidades a fin de determinar si el MMC es primario y/o secundario. La diferencia con la HPHA está basada en la eritrocitosis excesiva; dado que estos sujetos pueden cursar con HP se debe estimar la PAP en forma no invasiva o mediante el CCD.

\section{Conclusiones}

En condiciones de hipoxemia crónica y altitud mayor a 2,500 msnm la circulación pulmonar y el corazón derecho muestran cambios que sugieren ser las manifestaciones del proceso de la evolución hacia la adaptación a la altura.

Ambas entidades, la HP asociada a la HA y el MMC, comparten características clínicas, sin embargo, el nivel de $\mathrm{Hb}$ define el diagnóstico final entre ellos.

De acuerdo con la evidencia escrita aún existen dudas en cuanto al valor real de la presión pulmonar en nativos sanos de la altitud. Se requiere unificar criterios entre ambos consensos de la altitud y de la HP.

\section{Financiamiento}

La presente investigación no ha recibido ninguna beca específica de agencias de los sectores público, comercial, o sin ánimo de lucro.

\section{Conflicto de intereses}

Ninguno. 


\section{Responsabilidades éticas}

Protección de personas y animales. Los autores declaran que para esta investigación no se han realizado experimentos en seres humanos ni en animales.

Confidencialidad de los datos. Los autores declaran que han seguido los protocolos de su centro de trabajo sobre la publicación de datos de pacientes.

Derecho a la privacidad y consentimiento informado. Los autores declaran que en este artículo no aparecen datos de pacientes.

\section{Bibliografía}

1. Penaloza D, Arias Stella J. The heart and pulmonary circulation at high altitudes. Healthy highlanders and Chronic Mountain Sickness. Circulation. 2007;115(9):1132-14.

2. Barry PW, Pollard AJ. Altitude illness. BMJ. 2003;326(7395):915-19.

3. Beall, CM. Tibetan and Andean patterns of adaptation to high-altitude hypoxia. Hum Biol. 2000;72:201-28.

4. Martin D, Windsor J. From mountain to bedside: understanding the clinical relevance of human acclimatization to high-altitude hypoxia. Postgrad Med J. 2008;84(998):622-27.

5. Penaloza D. Effects of high-altitude exposure on the pulmonary circulation. Rev Esp Cardiol. 2012;65(12):1075-78.

6. Stuber T, Scherrer U. Circulatory adaption to long term high altitude exposure in Aymaras and Caucasians. Prog Cardiovasc Dis. 2010;52:534-39.

7. Wilkins Mr, Ghofrani H-A, Weissmann N, Aldeshev A, Zhao L. Pathophysiology and treatment of high-altitude pulmonary vascular disease. Circulation. 2015;132:582-90.

8. West JB. High-altitude medicine. Am J Respir Crit Care Med. 2012;12(186):1229-237.

9. Bartsch P, Swenson ER. Clinical practice: acute high-altitude illnesses. New Engl J Med. 2013:24(368):2294-302.

10. Villafuerte FC, Corante N. Chronic Mountain Sickness: Clinical aspects, etiology, management, and treatment. High Alt Med Biol. 2016;17(2):61-9

11. Maggiorini M, León Velarde F. High-altitude pulmonary hypertension: a pathophysiological entity to different diseases. Eur Respir J. 2003;22(6):1019-25.

12. León-Velarde F, Gamboa A, Chuquiza JA, Esteba WA, Rivera-Chira M, Monge $C$. Hematological parameters in high altitude residents living at 4355, 4660, and 5500 meters above sea level. High Alt Med Biol. 2000;1(2):97-104
13. Gassmann M, Mairbäurl H, Livshits L, Seide S, Hackbusch M, Monika Malczyk M, et al. The increase in hemoglobin concentration with altitude varies among human populations. Ann NY Acad Sci. 2019;1450:204-20.

14. Swenson ER. Hypoxic pulmonary vasoconstriction. High Alt Med Biol. 2013;14(2):101-8.

15. Murillo JC, Salinas SC, López MJJ, Villena CM. Función ventricular derecha en residentes nativos a gran altura. J Health Med Sci. 2020;6(2):113-22.

16. Stobdan T, Haddad GG. Commentary: Novel insight into the genetic basis of high altitude pulmonary hypertension in Kyrgyz highlanders. $J$ Lung Health Dis. 2019;3(2):29-30.

17. Grimminger J, Richter M, Tello K, Sommer N, Gall H, Ghofrani HA. Thin air resulting in high pressure: Mountain sickness and hypoxia-induced pulmonary hypertension thin air resulting in high pressure: Mountain sickness and hypoxia-induced pulmonary hypertension. Can Res J. 2017;2017:8381653.

18. León-Velarde F, Maggiorini M, Reeves JT, Aldashev A, Asmus I, Bernardo $\mathrm{L}$, et al. Consensus statement on chronic and subacute high altitude diseases. High Alt Med Biol. 2005;6(2):147-57.

19. McGoon M, Gutterman D, Steen V, Barst R, McCrory DC, Fortin TA, et al. Screening, early detection, and diagnosis of pulmonary arterial hypertension: ACCP evidence-based clinical practice guidelines. Chest. 2004;126(1 Suppl):14S-34S.

20. Aldashev AA, Sarybaev AS, Sydykov AS, Kalmyrzaev BB, Kim EV, Mamanova LB, et al. Characterization of high-altitude pulmonary hypertension in the Kyrgyz: association with angiotensin-converting enzyme genotype. Am J Respir Crit Care Med. 2002;166(10):1396-402.

21. Xu X-Q, Jing Z-C. High-altitude pulmonary hypertension. Eur Respir Rev. 2009;18(111):13-7.

22. Mandras SA, Mehta HS, Vaidya A. Pulmonary hypertension: A brief guide for clinicians. Mayo Clin Proc. 2020;95(9):1978-88.

23. Elia D, Caminati A, Zompatori M, Cassandro R, Lonati Ch, Luisi F, et al. Pulmonary hypertension and chronic lung disease: where are we headed? Eur Respir Rev. 2019;28(153):190065

24. Kojonazarov BK, Imanov BZ, Amatov TA, Mirrakhimov MM, Naeije R, Wilkins MR, et al. Noninvasive and invasive evaluation of pulmonary arterial pressure in highlanders. Eur Respir J. 2007;29(2):352-6.

25. Gopalan D, Blanchard D, Auger WR. Diagnostic evaluation of chronic pulmonary thromboembolic disease. Ann Am Thorac Soc. 2016;13(Suppl 3):S222-39.

26. Mullin CJ, Klinger JR. Chronic pulmonary thromboembolic disease. Heart Fail Clin. 2018;14(3):339-51.

27. Simonneau G, Montani D, Celermajer DS, Denton ChP, Gatzoulis MA, Krowka M, et al. Haemodynamic definitions and updated clinical classification of pulmonary hypertension. Eur Respir J. 2019;53(1):1801913.

28. Soria R, Egger M, Scherrer U, Bender N, Rimoldi SF. Pulmonary arterial pressure at rest and during exercise in chronic mountain sickness: a meta-analysis. Eur Respir J. 2019;53(6):1802040.

29. Naeije R. Pulmonary hypertension at high altitude. Eur Respir J. 2019;53(6):1900985.

30. Ge RL, Helun G. Current concept of chronic mountain sickness: pulmonary hypertension-related high-altitude heart disease. Wilderness Environ Med. 2001;12:190-4. 